\section{Novel antibody switching defects in human patients}

\author{
John P. Manis ${ }^{1,2}$ and Frederick W. Alt $t^{1,2,3,4}$ \\ ${ }^{1}$ The Children's Hospital, \\ ${ }^{2}$ Department of Genetics, Harvard Medical School, \\ ${ }^{3}$ Howard Hughes Medical Institute, and \\ ${ }^{4}$ The Center for Blood Research, Boston, Massachusetts, USA
}

Hyper-IgM syndrome (HIGM) is a primary immunodeficiency characterized by normal to elevated serum levels of $\operatorname{IgM}$ and low levels or the absence of IgG, IgA, and IgE. A new study (see related article on pages 136-142) characterizes HIGM type 4, a previously undocumented defect in antibody gene diversification caused by a selective block in classswitch recombination, providing significant insight towards understanding HIGM immunodeficiencies.

J. Clin. Invest. 112:19-22 (2003). doi:10.1172/JCI200319091.

B cells mediate humoral immunity by producing antibodies, which are comprised of immunoglobulin heavy $(\mathrm{IgH})$ and light (IgL) chains. The N-terminal variable region of antibodies is responsible for specific antigen binding. The $\operatorname{IgH}$ and IgL variable regions are encoded by exons that are assembled from germline $\mathrm{V}, \mathrm{D}$, and $\mathrm{J}$ gene segments during early lymphocyte development via $\mathrm{V}(\mathrm{D}) \mathrm{J}$ recombination. The $\mathrm{C}$-terminal end of the $\mathrm{IgH}$ chain is termed the $\mathrm{IgH}$ constant region $\left(\mathrm{C}_{\mathrm{H}}\right)$ and is responsible for determining both class of antibody and effector functions once it binds to a specific antigen (1). There are additional $C_{H}$ exons (referred to as $\mathrm{C}_{\mathrm{H}}$ genes lying in the several hundred kilobases downstream of the $V(D) J$ exons in the $\operatorname{IgH}$ locus (Figure 1a). The $\mathrm{V}(\mathrm{D}) \mathrm{J}$ is initially expressed in conjunction with the $\mathrm{C} \mu$ gene to form the $\mu$ heavy chain which, in

Address correspondence to: Frederick Alt, The Children's Hospital, 300 Longwood Avenue, Boston, Massachusetts 02115, USA. Phone: (617) 355-7290; Fax: (617) 738-0432;

E-mail: alt@enders.tch.harvard.edu.

Conflict of interest: The authors have

declared that no conflict of interest exists.

Nonstandard abbreviations used: immunoglobulin heavy ( $\operatorname{IgH})$; immunoglobulin light ( $\operatorname{IgL}) ; \operatorname{IgH}$ constant region $\left(\mathrm{C}_{\mathrm{H}}\right)$; class-switch recombination (CSR); switch region of the $\mu$ heavy chain ( $\mathrm{S} \mu$ region); somatic hypermutation (SHM); activation-induced deaminase (AID); hyper IgM syndrome (HIGM); uracil-DNA glycosylase (UNG); double-strand (DS); singlestrand (SS); ligation-mediated PCR (LM-PCR); double-strand break (DSB). association with IgL chain, forms IgM antibody (1). Once IgM+ $B$ cells engage antigen, two additional genetic alterations can occur to improve clearance of antibody-antigen complex or to increase binding affinity for antigen. In class switch recombination (CSR), the $\mathrm{C}_{\mathrm{H}}$ is switched from $\mu$ to a downstream $\mathrm{C} \gamma$, $\mathrm{C} \varepsilon$, or $\mathrm{C} \alpha$ gene, leading, respectively, to generation of IgG, IgE, or IgA isotypes with a corresponding change in antibody effector function (1). CSR involves deletional recombination between the switch region of the $\mu$ heavy chain ( $\mathrm{S} \mu$ region) upstream of $\mathrm{C} \mu$ and a similarly positioned $S$ region of a downstream $\mathrm{C}_{\mathrm{H}}$, allowing the $V(D) J$ exon to be juxtaposed to and expressed with a different $\mathrm{C}_{\mathrm{H}}$ gene (Figure 1a). A second antigendependent B cell genetic alteration, termed somatic hypermutation (SHM), involves introduction of point mutations at a high rate specifically into the IgH and IgL variable region exons, allowing for selection of a higher affinity antibody (2). Both CSR and SHM require transcription through target $S$ regions or $\mathrm{V}(\mathrm{D}) \mathrm{J}$ exons and also require activation induced deaminase (AID), an induced B cell-specific protein, showing that they share substantial mechanistic overlap, despite being unique processes $(1,3-5)$.

Inherited defects in class-switch recombination result in an immunodeficiency termed the hyper-IgM syndrome (HIGM), characterized by normal to elevated serum IgM but diminished levels of other IgH isotype classes. Mutations in the CD40-ligand or CD40-receptor impair proper B cell activation and are the cause of HIGM1 and HIGM3 respectively. Mutations in activation-induced deaminase (AID) underlie HIGM2. Both Aid-deficient mice and HIGM2 patients that lack functional AID have high levels of IgM but do not undergo CSR or SHM, showing that AID is absolutely necessary for these processes $(5,6)$. In addition, forced AID expression in nonlymphoid cells can generate CSR or SHM in reporter substrates, indicating that AID expression is sufficient to generate CSR and $\operatorname{SHM}(7)$, at least at low levels. The CSR block in AID-defective B cells is downstream of events leading to cellular activation and germline transcription (5), and most likely involves a defect in the generation of DNA lesions that initiate CSR (8). Most evidence favors a similar role for AID in SHM (4) (see below). AID has homology to RNA-editing cytidine deaminases, which led to the model that it may generate a novel recombinase involved with CSR or SHM via RNAediting (7). However, current evidence implicates DNA as the relevant AID substrate. In bacteria, AID-overexpression results in preferential mutation at $\mathrm{dC} / \mathrm{dG}$ nucleotide pairs, proposed to be a consequence of DNA cytidine deaminase activity generating G-U mispairs and triggering the uracil-DNA glycosylase (UNG) DNA repair pathway (9). In this context, Ung-deficient mice exhibit a substantial defect in both CSR and in SHM, indicating that this repair pathway may function downstream of AID in both processes (10). Most recently, biochemical studies showed that AID has DNA cytidine deaminase activity on single-strand (SS), but not doublestrand (DS) DNA in vitro (11-13); and that AID could be targeted to DS DNA via transcription (12). Moreover, genetargeted $\mathrm{S}$ region mutations in mice provided in vivo evidence for the model that transcriptionally-generated SS DNA structures in $S$ regions are physiological CSR substrates, and other studies showed that relevant structures (R-loops) form in vivo in the context of $S$ region transcription $(14,15)$. Therefore, transcription through $\mathrm{S}$ regions 
appears to lead to generation of SS DNA substrates for AID, which then serves to initiate CSR via DNA cytidine deamination (Figure 1b). In addition, bacterial studies have implicated transcription as generating SS DNA substrates for AID in the context of mutation (16).

\section{CSR defects downstream of AID activity}

There are still many unsolved questions regarding CSR and SHM, including why deaminated cytidines are repaired in an error-prone fashion, and what specifies a CSR versus SHM fate for deaminated DNA sequences. In addition, the mechanism by which AID is targeted to variable region sequences during SHM appears distinct from that which targets it to $S$ regions and may involve additional factors to generate SS DNA substrates (12). Also, little is known about the factors involved downstream of AID in CSR, particularly in the context of the precise nature of the differential repair pathways utilized and also with respect to the factors that mediate synapsis of two recombining $S$ regions. Given these clear differences in the processes, potential defects in postAID-dependent steps of CSR and SHM might be expected to lead to the generation of B cells with differentially impaired CSR and/or SHM. In this issue of the JCI, Imai et al. (17) now, in fact, describe a novel subset of HIGM patients (termed HIGM4) who have defective CSR but normal SHM. In HIGM4 patient B cells, CSR events upstream of AID activation, including germline $\mathrm{C}_{\mathrm{H}}$ transcription, were normal; a ligation-mediated PCR assay detected blunt DSB's in the $S \mu$ region suggesting that AID initiated the process. However, there was no production of mature $\mathrm{IgH}$ transcripts or secreted antibodies indicating that CSR was defective in the HIGM4 B cells despite both AID and S region activation. Strikingly, SHM in these patients was normal in both numbers of cells mutated, as well as in over-

a $\quad \mathrm{V}(\mathrm{D})$

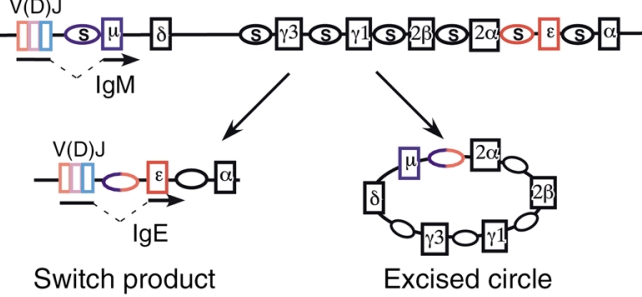

b $S$ regions rendered accessible by transcription and $\mathrm{R}$ loop formation

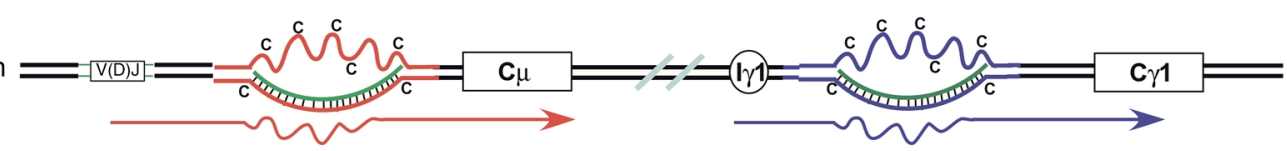

Cytidine deamination of single-stranded DNA by AID
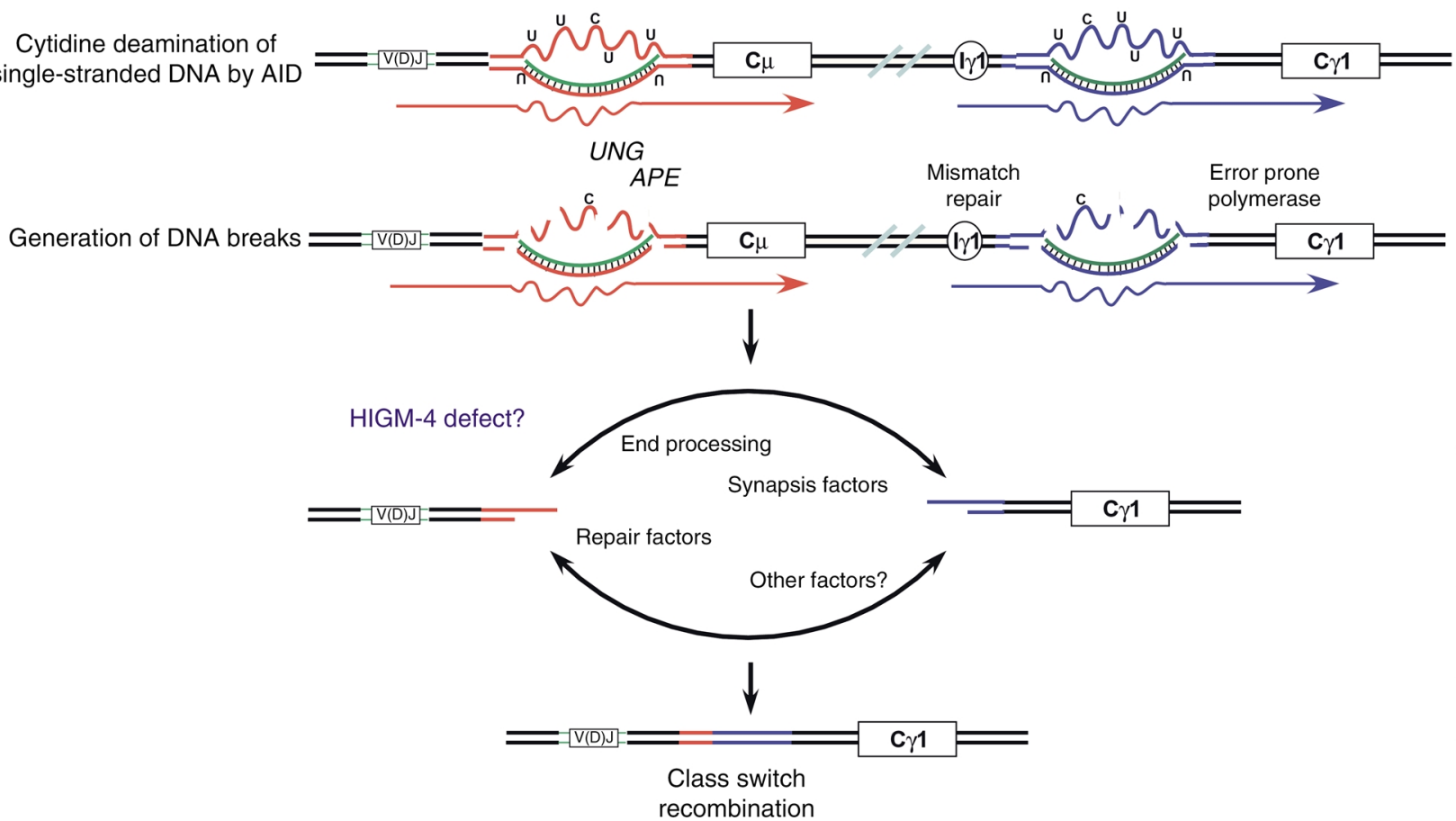

Figure 1

(a) The rearranged $V(D)$ J exon is located immediately upstream of the $\mu$ constant region with all other classes of murine constant region genes lying downstream, with each (except $C \delta$ ) preceded by repetitive DNA sequences (termed switch (S) regions) that are between 1-12 kb in length. (b) The variable region exon or $\mathrm{S}$ regions targeted for modification are rendered accessible by transcription. Secondary structures, shown here for $\mathrm{S}$ regions, are formed and allow for direct SS DNA modification by AID, resulting in dU/dG mismatches. Differential sensing, processing, and resolution of these mismatches results in distinct outcomes for CSR or SHM. 
all mutation load. Finally, HIGM4 B cells proliferated normally following stimulation for CSR indicating that the defect is not associated with overall cellular survival, as is seen in certain murine NHEJ-deficient B cells (1). Thus, these findings imply a selective defect in CSR versus SHM that occurs downstream of AID in HIGM4 syndrome, indicating that HIGM4 $\mathrm{B}$ cells may provide an important reagent for further elucidation of events specific to CSR. In this regard, it is not known whether all HIGM4 mutations affect the same factor or represent several different defects that manifest a common phenotype.

\section{Selective targeting of CSR versus SHM}

What processes may distinguish CSR and SHM and, thereby, be potential targets of HIGM4 mutations? Although DNA cytidine deamination is common to both processes, CSR and SHM can occur differentially in B cells, generate distinct products, and are likely resolved by separate repair pathways $(18,19)$. Thus, AID is specifically targeted to the appropriate Ig sequence (V[D]J vs. S regions) with subsequent processing of the deaminated cytidines occurring in such a way as to promote either CSR or SHM. SHM and CSR probably generate distinct secondary structures that are targets for AID. S regions, due to their unique base composition, can form transcription-dependent higher order structures, such as R loops, which provide SS DNA targets for AID. However, such structures are not expected to occur in SHM target sequences; in this process, a transient SS DNA transcription bubble in the variable region exon may help provide an AID substrate (16, 18). In this regard, AID may be associated in different factor complexes at target $V(D) J$ or $S$ region sequences and serve to recruit different CSR- or SHMspecific AID-cofactors $(3,19)$. While it seems likely that most putative cofactors of this type would be intact in HIGM4 B cells as $S$ region breaks were detected upon activation, there is still potential for such a CSR-specific factor. Thus, HIGM4 cells were examined only for $S \mu$ breaks and not for downstream S region breaks; leaving open the possibility of a defect in downstream $S$ region targeting factor. Also, the differential fate of AID-induced dU residues at $\mathrm{V}(\mathrm{D}) \mathrm{J}$ exons or $\mathrm{S}$ regions may be linked to aspects of mismatch recognition (18, 20). In this regard, $S$ regions may allow generation of higher density of AID-dU modifications potentially leading to a greater likelihood of generating staggered DNA breaks on both DNA strands by downstream damage recognition pathways and, thereby, engage a different subset of factors than SHM.

\section{Differential processing and repair}

With respect to potential downstream events that may distinguish CSR from SHM, there are other potentially involved processes/factors worth considering. The nature of the final products of SHM (point mutations) and of CSR (joining of two $S$ regions) suggests involvement of a distinct repair pathway for each process. CSR requires synapsis of two $S$ regions that may be separated by over $100 \mathrm{~kb}$, with the deletion of the intervening DNA. Putative synapsis factors required for CSR may not be involved in SHM as the latter process involves nucleotide changes made over relatively short sequences. Absence of such a putative CSR-synapsis factor might manifest defects similar to those seen in HIGM4. In this regard, it is notable that cell cycle checkpoint/repair deficiencies (e.g., ataxia-telangiectasia mutated, Nijmegen breakage syndrome) affect CSR (21). In addition, deficiencies in the Histone H2AX protein substantially impairs CSR (7) and may lead to S region translocations (C. Bassing and F.W. Alt, unpublished observations). While the mechanism of these defects is not clearly understood, there is a possibility that one or all may be directly or indirectly involved in synapsis. It is also worth noting that CSR requires an intact NHEJ pathway of DNA DSB repair, that along with other evidence, has led to arguments that CSR involves DSB's that ultimately are joined by NHEJ $(1,19)$. In contrast, SHM does not appear to involve NHEJ (19). While it seems unlikely that defects in NHEJ would underlie the HIGM4 syndrome, as these would also lead to a SCID phenotype due to defective $\mathrm{V}(\mathrm{D}) \mathrm{J}$ recombination, it is worth noting that absence of a particular NHEJ factor, DNA-dependent protein kinase catalytic subunit, preferentially affects CSR versus SHM (19).

Whatever its basis, the newly described HIGM4 defect once again illustrates the power of defining human disease mechanisms with respect to elucidating basic physiological processes. Thus, further characterization of HIGM4 mutations may well provide a potent means to further elucidate processes involved in CSR events downstream of AID, which is now one of the major unsolved problems in this field.

\section{Acknowledgments}

We thank Ming Tian, Jayanta Chaudhuri, and Katrin Chua for helpful discussions. This work was supported by NIH grant AI31541 (F.W. Alt) and Lymphoma Research Foundation Grant (J.P. Manis). F.W. Alt is an Investigator for the Howard Hughes Medical Institute.

1. Manis, J.P., Tian, M., and Alt, F.W. 2002. Mechanism and control of class-switch recombination. Trends Immunol. 23:31-39.

2. Papavasiliou, F.N., and Schatz, D.G. 2002. Somatic hypermutation of immunoglobulin genes: merging mechanisms for genetic diversity. Cell. 109:S35-S44.

3. Faili, A., et al. 2002. AID-dependent somatic hypermutation occurs as a DNA single-strand event in the BL2 cell line. Nat. Immunol. 3:815-821.

4. Chua, K.F., Alt, F.W., and Manis, J.P. 2002. The function of AID in somatic mutation and class switch recombination: upstream or downstream of DNA breaks. J. Exp. Med. 195:F37-F41.

5. Muramatsu, M., et al. 2000. Class switch recombination and hypermutation require activationinduced cytidine deaminase (AID), a potential RNA editing enzyme. Cell. 102:553-563.

6. Revy, P., et al. 2000. Activation-induced cytidine deaminase (AID) deficiency causes the autosomal recessive form of the Hyper-IgM syndrome (HIGM2). Cell. 102:565-575.

7. Honjo, T., Kinoshita, K., and Muramatsu, M. 2002. Molecular mechanism of class switch recombination: linkage with somatic hypermutation. Annu. Rev. Immunol. 20:165-196.

8. Petersen, S., et al. 2001. AID is required to initiate $\mathrm{Nbs} 1 /$ gamma-H2AX focus formation and mutations at sites of class switching. Nature. 414:660-665.

9. Petersen-Mahrt, S.K., Harris, R.S., and Neuberger, M.S. 2002. AID mutates E. coli suggesting a DNA deamination mechanism for antibody diversification. Nature. 418:99-103.

10. Rada, C., et al. 2002. Immunoglobulin isotype switching is inhibited and somatic hypermutation perturbed in UNG-deficient mice. Curr. Biol. 12:1748-1755.

11. Dickerson, S.K., Market, E., Besmer, E., and Papavasiliou, F.N. 2003. AID Mediates Hypermutation by Deaminating Single Stranded DNA. J. Exp. Med. 197:1291-1296.

12. Chaudhuri, J., et al. 2003. Transcription-targeted DNA deamination by the AID antibody diversification enzyme. Nature. 422:726-730.

13. Bransteitter, R., Pham, P., Scharff, M.D., and Goodman, M.F. 2003. Activation-induced cytidine deaminase deaminates deoxycytidine on a single-stranded DNA but requires the action of 
RNase. Proc. Natl. Acad. Sci. U. S. A. 100:4102-4107.

14. Shinkura, R., et al. 2003. The influence of transcriptional orientation on endogenous switch region function. Nat. Immunol. 4:435-441.

15. Yu, K., Chedin, F., Hsieh, C.L., Wilson, T.E., and Lieber, M.R. 2003. R-loops at immunoglobulin class switch regions in the chromosomes of stimulated B cells. Nat. Immunol. 4:442-451.

16. Ramiro, A.R., Stavropoulos, P., Jankovic, M., and Nussenzweig, M.C. 2003. Transcription enhances
AID-mediated cytidine deamination by exposing single-stranded DNA on the nontemplate strand. Nat. Immunol. 4:452-456.

17. Imai, K., et al. 2003. Hyper-IgM syndrome type 4 with a B lymphocyte-intrinsic selective deficiency in Ig class-switch recombination. J. Clin. Invest. 112:136-142. doi:10.1172/JCI200318161.

18. Storb, U., and Stavnezer, J. 2002. Immunoglobulin genes: generating diversity with AID and UNG. Current Biology. 12:725-727.
19. Kenter, A.L. Class-switch recombination: after the dawn of AID. 2003. Curr. Opin. Immunol. 15:190-198.

20. Martin, A., and Scharff, M.D. 2002. AID and mismatch repair in antibody diversification. Nat. Rev. Immunol. 2:605-614.

21. Pan, Q., et al., 2002. Alternative end joining during switch recombination in patients with ataxia-telangiectasia. 2002. Eur. J. Immunol. 32:1300-1308.

\title{
DNA vaccines and apoptosis: to kill or not to kill?
}

\author{
Wolfgang W. Leitner and Nicholas P. Restifo
}

National Cancer Institute, National Institutes of Health, Bethesda, Maryland, USA

\begin{abstract}
The apoptotic machinery has become the latest target of vaccinologists attempting to improve the efficacy of DNA vaccines. While workers have previously sought to induce apoptotic death in transfected DCs as a means to activate immunity, a new approach (see related article on pages 109-117) instead seeks to delay apoptosis in host DCs after DNA vaccination.
\end{abstract}

J. Clin. Invest. 112:22-24 (2003). doi:10.1172/JCI200319069.

The honeymoon period that vaccinologists had with the new technology of DNA immunization is over. It ended with the realization that DNA vaccines were not as effective as hoped against the most serious threats such as HIV or cancer. Disappointing results from ongoing preclinical work and from clinical trials have put a serious damper on the enthusiasm that characterized the early days of DNA vaccines. It nevertheless seems to us that an overwhelming set of theoretical and practical advantages justify a redoubling of effort to get DNA vaccines to work effectively in humans. This is particularly the case when the menace of bioterrorism looms ever larger, and threats of new epidemics caused by emerging infectious diseases, such as Severe Acute Respiratory Syndrome, seem to be materializing. It is, needless to say, critically important to have vaccine vectors that can rapidly be engineered and administered to large numbers of people using a pathogen's genetic information.

\footnotetext{
Address correspondence to: Nicholas $P$. Restifo, Surgery Branch, National Cancer Institute, Building 10, Room 2B46, National Institutes of Health, Bethesda, Maryland 20892, USA. Phone: (301) 496-4904; Fax: (301) 402-0922; E-mail: restifo@nih.gov. Conflict of interest: The authors have declared that no conflict of interest exists.
}

Nucleic acid vaccines represent such a vaccine vector - the requisite cultivation and expansion of new pathogens for the creation of a live attenuated or killed vaccine is of course not necessary when all one needs for construction of a vaccine is the bug's genetic identity.

For this reason, research on DNA vaccines has moved to its second phase with the emphasis now on improving immunogenicity and efficacy (reviewed in ref. 1). This includes: (i) improved DNA plasmids used as vectors in an attempt to enhance antigen expression and focus antigen targeting; (ii) better delivery systems for more efficient transfection of cells in vivo; and (iii) the development of molecular adjuvants to enhance immune responses to the inoculum, including the codelivery of cytokine (2) or other adjuvant molecules (3).

The drive to improve DNA vaccine function is fueled by the consensus that DNA vaccines may be immunologically benign, that is to say, they are simply not carrying enough of the signals necessary to trigger a strong innate immune response. While immunostimulatory DNA sequences (CPG motifs) are believed to be primarily responsible for the adjuvant properties of prokaryotic DNA (4), the adjuvant capacity of CPG that naturally occur on plasmids may not be sufficient for many applications. This is especially true when dealing with weakly immunogenic antigens or selfantigens, as is the case with cancer. The issue of immunostimulatory DNA is further complicated by the identification of species-specific requirements for these motifs. Thus, there is an urgent need for more robust and universally applicable adjuvant strategies.

\section{Induction of apoptosis enhances DNA vaccine immunogenicity}

The immunostimulatory properties of apoptotic death have been debated intensively in recent years (5-9). It appears that the controversy over whether apoptosis or necrosis are either immunostimulatory or immunosuppressive were - at least in part - due to the misguided view that apoptotic death came in a single variety. Based on early descriptions, apoptosis was defined as a particular kind of cell death occurring in the absence of inflammation with predictable and invariable lack of immune stimulation. More recent studies have made it clear that apoptotic death can be triggered by a wide variety of mechanisms, which depending on the trigger can be accompanied by the production and release of various factors that help the immune system make a decision about the handling of the dead cells (10). Thus, apoptosis has been redefined as a particular set of defined molecular events with myriad variations.

Various reports have shown the immunogenicity of antigenic material associated with dead or dying cells ( 7 , 11) and several studies have applied these findings in their effort to enhance DNA vaccine efficacy. Workers have codelivered genes for proapoptotic molecules with DNA vaccines to specifically induce apoptosis in transfected cells. For example, $\mathrm{CD}^{+}$and $\mathrm{CD}^{+} \mathrm{T}$ cell responses were improved when the genes for mutated caspases 2 or 3 were coinjected with the antigen-carrying 